\title{
Carrier Allocation for Hybrid Satellite-Terrestrial Backhaul Networks
}

\author{
Eva Lagunas, Sina Maleki, Lei Lei, Christos Tsinos, Symeon Chatzinotas, Björn Ottersten \\ Interdisciplinary Centre for Security, Reliability and Trust (SnT) \\ University of Luxembourg, 29 Av. J.F. Kennedy, L-1855, Luxembourg
}

\begin{abstract}
In this paper, we consider the problem of carrier allocation in Hybrid Satellite-Terrestrial Backhaul (HSTB) networks, where the satellite segment and the terrestrial backhaul network are integrated in a seamless manner. To enhance the overall spectral efficiency of the backhaul network, we consider that both terrestrial and satellite segments operate in the 17.719.7 GHz band, where the sharing between Fixed-Service (FS) microwave links and satellite communications is allowed. Due to sharing the same spectrum, both systems are subject to interference constraints which should be properly taken into account in the carrier allocation algorithm design. Focusing on sum-rate as the key performance indicator, we formulate the underlying optimization problem which tends to be NP-hard. To overcome this hurdle, we propose to tackle the satellite and the terrestrial carrier allocation in a sequential manner. The proposed algorithm is compared and validated using numerical results considering a realistic topology and system parameters.
\end{abstract}

\section{INTRODUCTION}

The deployment of the upcoming fifth generation of cellular systems $(5 \mathrm{G})$ has posed numerous challenges, mainly in terms of supporting very high data rates with low endto-end delays [1], [2]. The expected traffic growth is posing significant burdens on the wireless backhaul network [3]. In fact, backhauling, which refers to the communication network between the base stations and the core network, has been identified as a key challenge for the $5 \mathrm{G}$ deployment [4]. With the understanding that the backhaul portion of the network is the bottleneck of the entire network, cellular operators are searching for new ways to meet the growing traffic demand but at the same time alleviate the backhaul congestion by reusing the available resources as much as possible.

Satellite communications is one of the emerging wireless backhaul solutions which has been identified as a key technology to narrow the large and growing gap within the digital divide that is prevalent in many countries nowadays [5]. More precisely, the satellite system can complement terrestrial backhaul infrastructure in all geographical regions including rural and urban areas. Although there are significant advances in terrestrial technologies, there are still many places which are inaccessible for the current terrestrial backhaul network infrastructure [6]. For the remote rural areas where target capacity cannot be met with terrestrial backhaul system, or where it is too costly, or simply where it is not suitable to expand the current infrastructure, satellite communications offer a viable solution. Regarding urban areas, the satellite segment can provide a backup solution to the terrestrial backhaul links in case of failure or for load balancing in dense

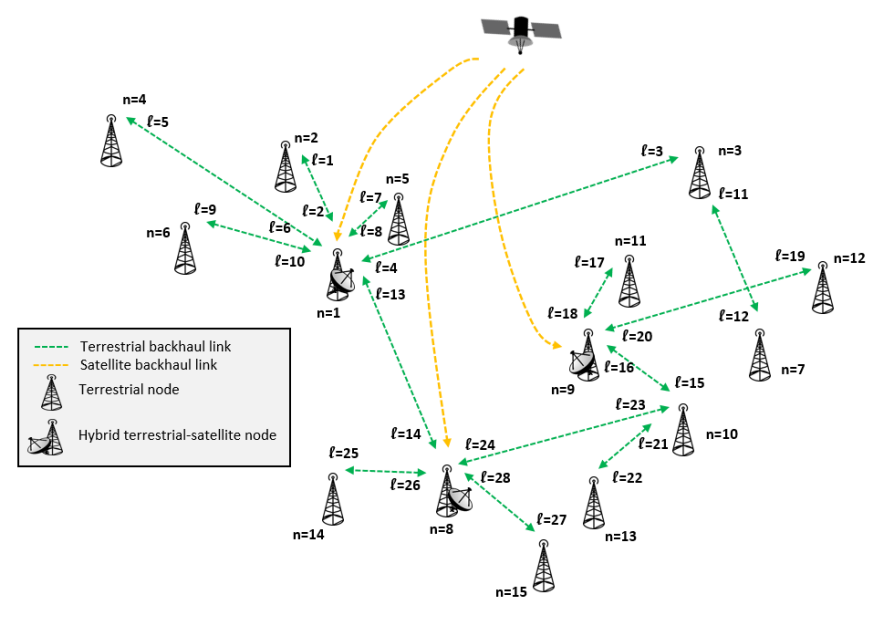

Fig. 1: Hybrid satellite-terrestrial backhaul network topology in the $17.7-19.7 \mathrm{GHz}$ band.

areas with high peak traffic demands, e.g. in a sport stadium where big events take place.

Recently, the concept of Hybrid Satellite-Terrestrial Backhaul (HSTB) network has been developed in different research projects such as VITAL (VIrtualized hybrid satelliteTerrestriAl systems for resilient and fLexible future networks) [7] or SANSA (Shared Access Terrestrial-Satellite Backhaul Network enabled by Smart Antennas) [8]. In the considered HSTB network, the satellite system is no longer seen as a competitor component but as a part of a seamless integrated system, where the terrestrial backhaul network takes advantage from specific properties of the satellite segment to improve resiliency to link failures and congestion, and/or for traffic offloading.

The peculiarity of SANSA is that terrestrial and satellite segments share the same spectrum in order to enhance the overall spectrum efficiency. In this paper, we focus on one of the selected scenarios in SANSA: the Satellite-to-Earth backhauling links, which operate in the 17.7-19.7 GHz band together with the terrestrial backhaul network. In the considered scenario, which is depicted in Fig. 1, the interference from terrestrial backhauling transmitters to the satellite backhauling terminals needs to be taken into account in order to guarantee operation of the satellite segment of the system. Moreover, in this paper we consider aggressive frequency reuse schemes 
within the terrestrial segment which generate terrestrial intrasystem interference as well.

Hence, it is crucial to properly design the frequency allocation of the HSTB with the aim of sharing the available and limited spectrum resources between terrestrial and satellite segments as efficiently as possible. Carrier frequency allocation for cognitive satellite communications with incumbent terrestrial networks has been considered in [9], [10], where the satellite carrier allocation is designed for a given terrestrial frequency allocation. However, research effort on carrier allocation for HTSB networks is still in its infancy [11]. In [11], a descentralized joint resource allocation and routing algorithm is proposed in the framework of hybrid terrestrialsatellite networks where both segments operate in exclusive spectrum bands.

In this paper, we investigate the carrier allocation for HTSB networks where the terrestrial and the satellite segments share the same spectrum bands. More precisely, we formulate the joint carrier allocation optimization problem focusing on sumrate maximization and assuming fixed trasmit powers. We show that the latter is a NP-hard problem due to the coupling between the satellite and terrestrial carrier allocation. To overcome this hurdle, we propose to optimize each part of the HTSB network in a sequential manner, so that the first optimization step provides the satellite carrier allocation that is taken into account in the second optimization step which tackles the terrestrial part of the HTSB network.

The remainder of this paper is organized as follows. In Section II we present the HSTB system model. Section III focuses on the proposed carrier allocation strategy. In Section IV we provide supporting results based on numerical data. Finally, Section V concludes the paper.

\section{SyStem ModeL}

We consider a multi-hop wireless backhaul network composed of several terrestrial stations. Some of them are equipped with a satellite dish antenna and, therefore, have the possibility to receive backhaul traffic through the satellite network. The considered scenario is illustrated in Fig. 1, where the illustrated terrestrial topology is part of the true backhaul network of Finland, which was obtained from the Finnish regulator. We assume that all the nodes in the HSTB network have access to the core through multi-hop wireless links, either through the terrestrial segment or the satellite segment of the network.

\section{A. Terrestrial Segment Model}

Let us consider a hybrid backhaul network with $N$ terrestrial nodes indexed by $n=1, \ldots, N$, which can send, receive and relay backhaul traffic. We consider the terrestrial nodes to be interconnected through $L$ unidirectional communication links, indexed by $l=1, \ldots, L$, as illustrated in Fig. 1. Following the notation in the literature [12], [13], we represent the set of terrestrial links that are outgoing from node $n$ with $\mathcal{O}(n)$ and the set of terrestrial links that are incoming to node $n$ with $\mathcal{I}(n)$.
Let us assume that the $L$ terrestrial links operate in the 17.719.7 $\mathrm{GHz}$ band. We assume that the 17.7-19.7 $\mathrm{GHz}$ band is divided into $K$ equally sized frequency carriers of bandwidth size $B_{t}$, which represent a standard bandwidth supported by the terrestrial system. For the sake of clarity, we assign a carrier identification number to each of the $K$ frequency carriers, namely $k=1, \ldots, K$. We assume $L>K$, which is the general and most challenging case.

Let $\mathbf{a}_{t} \in \mathbb{R}^{L}$ be the terrestrial carrier allocation vector, whose elements $a_{t}(i) \in[1, K]$ contain the terrestrial carrier identification number of the carrier that has been assigned to the $i$-th terrestrial link. Note that for implementation issues, only one carrier should be assigned to each link. Additionally, full-duplex scenarios in which the same carrier is used for transmission and reception at the same terrestrial station should be avoided. The latter constraint can be mathematically expressed as follows:

$$
\mathbf{a}_{t}(j) \neq \mathbf{a}_{t}(i), \quad j \in \mathcal{I}(n), i \in \mathcal{O}(n), \forall n .
$$

On the other hand, one carrier can be simultaneously assigned to multiple links. This goes inline with the current trend of targeting aggressive frequency reuse schemes, which are expected to increase the spectrum efficiency and network capacity at the expense of increased interference levels. From the total $K$ carriers, the carrier allocation algorithm chooses $K^{\prime} \leq K$ to design the terrestrial backhaul network.

Regarding the interference modeling, the terrestrial intrasystem interference signal level seen by the $l$-th terrestrial link operating at the $k$-th carrier can be expressed as follows:

$$
i_{t}^{l}(k)=\sum_{\substack{i \in \mathcal{T}(l) \\ i \neq l}} P_{\mathrm{tx}}^{\mathrm{TER}}(i) \cdot G_{\mathrm{tx}}^{\mathrm{TER}}\left(\theta_{i, l}\right) \cdot h(i, k, l) \cdot G_{\mathrm{rx}}^{\mathrm{TER}}\left(\theta_{l, i}\right)
$$

where,

- $\mathcal{T}(l)$ : Set of terrestrial links sharing the same frequency carrier with the $l$-th terrestrial link,

- $P_{\mathrm{tx}}^{\mathrm{TER}}(i)$ : Transmit power of the $i$-th link' transmit station,

- $G_{\mathrm{tx}}^{\mathrm{TER}}(\theta)$ and $G_{\mathrm{rx}}^{\mathrm{TER}}(\theta)$ : Gain of the terrestrial transmitting/receiving antenna at an offset angle $\theta$. The radiation pattern can be obtained from ITU-R F.1245-2,

- $\theta_{i, l}$ : Offset angle (from the boresight direction) of the $i$ th link transmit antenna in the direction of the $l$-th link receiver antenna,

- $h(i, k, l)$ : Propagation loss considering free space path loss and diffraction loss computed according to the Bullington model described in ITU-R P.526-13 between the $i$-th link transmit station and the $l$-th link receiver station when operating at the $k$-th carrier.

According to (2), the Signal-to-Interference plus Noise Ratio (SINR) of the $l$-th terrestrial backhaul link operating at the $k$-th carrier can be computed as follows,

$$
\operatorname{SINR}_{\mathrm{t}}(l, k)=\frac{P_{\mathrm{tx}}^{\mathrm{TER}}(l) \cdot G_{\mathrm{tx}}^{\mathrm{TER}}(0) \cdot h(l, k, l) \cdot G_{\mathrm{rx}}^{\mathrm{TER}}(0)}{i_{t}^{l}(k)+N_{t}},
$$

where $N_{t}$ is the noise thermal power. 


\section{B. Satellite Segment Model}

In this paper, we focus on the Satellite-to-Earth transmission direction. Let us consider $M \leq N$ terrestrial nodes equipped with satellite dish antennas. Note that in the satellite segment, the number of links is equal to the number of hybrid satelliteterrestrial nodes.

The $M$ satellite links operate in the same $17.7-19.7 \mathrm{GHz}$ band than the terrestrial segment. We assume that the 17.7$19.7 \mathrm{GHz}$ band is divided into $C$ equally sized frequency carriers of bandwidth size $B_{s}$, which represent the satellite forward carrier bandwidth. For the sake of clarity, we assigned a carrier identification number to each of the $C$ frequency carriers, namely $c=1, \ldots, C$. Similarly, from the total $C$ carriers, the carrier allocation algorithm chooses $C^{\prime} \leq C$ to design the satellite backhaul network.

Let $\mathbf{a}_{s} \in \mathbb{R}^{M}$ be the satellite carrier allocation vector, whose elements $a_{s}(i) \in[1, C]$ contain the carrier identification number of the satellite carrier that has been assigned to the $i$-th satellite link. The forward satellite links work on a single carrier communication mode and, thus, they can only be assigned one carrier frequency and this should not be shared with others satellite links. Based on this discussion, $C^{\prime}=M$ and the following constraint shall be considered:

$$
a_{s}(i) \neq a_{s}(j) \quad \text { for } \quad i, j=1, \ldots, M, i \neq j
$$

which ensures that a single carrier is not assigned to multiple links.

On the other hand, and due to the spectrum sharing assumption, satellite links will be affected by the interference caused by the terrestrial backhaul links. Let us denote $\mathcal{S}(m)$ as the set of terrestrial links that share the same carrier frequency than the $m$-th satellite link. The interference level seen at the $m$-th satellite link operating at the $c$-th carrier can be written as,

$$
i_{s}^{m}(c)=\sum_{i \in \mathcal{S}(m)} P_{\mathrm{tx}}^{\mathrm{TER}}(i) \cdot G_{\mathrm{tx}}^{\mathrm{TER}}\left(\theta_{i, m}\right) \cdot h(i, c, m) \cdot G_{\mathrm{rx}}^{\mathrm{SAT}}\left(\theta_{m, i}\right)
$$

where $G_{\mathrm{rx}}^{\mathrm{SAT}}(\theta)$ is the gain of the satellite dish receiving antenna at an offset angle $\theta$. The radiation pattern of the dish antenna can be obtained from ITU-R S.465-6. Variable $h(i, c, m)$ denotes the propagation loss between the transmit antenna of the $i$-th terrestrial link and the satellite terminal receiver of the $m$-th satellite link when operating at the $c$-th carrier. The latter is computed taking into account both path and diffraction losses. In (5), we assume that the interfering signal falls within the victim bandwidth. If the spectra do not overlap completely, then a compensation factor of $B_{\text {overlap }} / B_{s}$ is applied, where $B_{\text {overlap }}$ stands for the portion of the interfering signal spectral density within the receive modem filter bandwidth given by $B_{s}$.

According to (5), the SINR of the satellite backhaul links can be computed as follows,

$$
\operatorname{SINR}_{\mathrm{s}}(m, c)=\frac{P_{s} \cdot G_{s} \cdot h_{s}(m, c) \cdot G_{\mathrm{rx}}^{\mathrm{SAT}}(0)}{i_{s}^{m}(c)+I_{\mathrm{co}}+N_{s}},
$$

where $P_{s}$ refers to the satellite transmit power, $G_{s}$ denotes the satellite antenna gain, $h_{s}(m, c)$ denotes the satellite channel gain of the $m$-th satellite link operating at the $c$-th carrier (only free space path loss is considered for the satellite links), $I_{\mathrm{co}}$ is the co-channel interference due to the use of multibeam satellite, and $N_{s}$ is the thermal noise power seen at the satellite dish antenna.

\section{Proposed Carrier Allocation Algorithms}

The goal of this paper is to design jointly the terrestrial and satellite carrier assignment for which the interference impact on both the terrestrial and satellite link performance is minimal. The allocation processing is assumed to take place in a centralized controller that has access to the overall network planning information. To be more specific, in this section, we will focus on the maximization of the links' sum-rate, which is one of the most popular figure of merit used for spectrum management [10]. In this paper, we consider that the transmit power is fixed.

The corresponding sum-rate maximization problem is formulated in (7):

$$
\begin{array}{|ll|}
\max _{\mathbf{a}_{t}, \mathbf{a}_{s}} & \sum_{j=1}^{L} R_{t}(j)+\sum_{j=1}^{M} R_{s}(j) \\
\text { s.t. } & a_{t}(j) \in[1, K], j=1, \ldots, L \\
& a_{s}(j) \in[1, C], j=1, \ldots, M \\
& a_{s}(i) \neq a_{s}(j) i, j=1, \ldots, M, i \neq j \\
& a_{t}(j) \neq a_{t}(i), \quad j \in \mathcal{I}(n), i \in \mathcal{O}(n), \forall n
\end{array}
$$

where $R_{t}(j)=B_{t} \log _{2}\left(1+\operatorname{SINR}_{t}\left(j, \mathbf{a}_{t}\right)\right)$ and $R_{s}(j)=$ $B_{s} \log _{2}\left(1+\operatorname{SINR}_{s}\left(j, \mathbf{a}_{t}, \mathbf{a}_{s}\right)\right)$.

Clearly, the considered joint carrier allocation problem of terrestrial and satellite segment is intractrable due to the nonlinear coupling between each other. This is because the terrestrial allocation directly disturb the sum-rate of the satellite backhaul network. Even the sum-rate maximization of only terrestrial links remains intractable since the link allocation per carrier amounts to determine which terrestrial link should be grouped into an interfering channel such that sum-rate is maximized, and this problem is in general NP-hard [14]. More specifically, solving (7) would require to take into account all possible carrier combinations, which is computationally prohibitive in general.

To handle the problem, in this paper we make use of decomposition methods [12] in which the coupled problem is split into several small sub-problems. More precisely, in this paper we first deal with the carrier assignment for the satellite backhaul network and, on a second step and assuming the resulting satellite segment allocation, we design a sub-optimal carrier assignment for the terrestrial part of the network. As discussed before, the latter is not a tractable problem and, thus, finding the optimal solution is very challenging. Therefore, we propose an algorithm to solve the terrestrial assignment iteratively. 
The order in which each part of the hybrid network is optimized first is driven by the degrees of freedom of the resource allocation problem, which in our case is higher for the terrestrial network since it has more flexibility to adapt to the existing spectral environment.

\section{A. Step 1: Carrier Allocation for the Satellite Backhaul Net- work}

Given the single carrier transmission of the satellite segment, the satellite spectrum usage is dictated by the number of satellite links. Therefore, the carrier allocation of the satellite segment reduced to find the combination of $M$ satellite links with $C^{\prime}$ satellite carriers, where $M=C^{\prime}$. In this first step, we proceed as if there were no terrestrial network and, thus, the satellite carrier allocation is done based on each satellite link SINR conditions, as in [10], [15]. In other words, the sum-rate of the satellite system is maximized by selecting the carrier that provides higher SINR to each satellite link subject to (4), where the SINR is computed as in (6) with $i_{s}^{m}(c)=0$, $m=1, \ldots, M, c=1, \ldots, C$. This carrier-link assignment problem can be very efficiently solved with the Hungarian method [16].

\section{B. Step 2: Carrier Allocation for the Terrestrial Backhaul Network}

The terrestrial carrier allocation optimization problem is a full search space problem, which means searching for the best among all the possible combinations of carrier assignments. This assignment process is tedious and time-consuming. In this section, we propose an iterative algorithm that solve the carrier assignment in a sequential manner so that at each step the search space is reduced.

To maximize the sum-rate of both satellite and terrestrial segments, terrestrial intra-system interference should be minimized so that the SINR of the terrestrial links, given in (3), is maximized. This means that the optimal carrier allocation will tend to be carrier hungry. That is, if the channel condition on an empty carrier is acceptable for a particular link, this link should be allocated to the empty carrier instead of being allocated to one carrier that is being used by other links. On the other hand, the terrestrial interference seen at the satellite receivers, i.e. inter-system interference, should be minimized. In other words, the terrestrial carrier allocation should not only maximize the SINR of the terrestrial links but also maximize the SINR of the satellite links, which is given in (6). Therefore, the terrestrial assignment should take into account both intrasystem and inter-system interference.

Regarding the terrestrial intra-system interference, let us define the SINR matrix of the terrestrial links as follows,

$$
\operatorname{SINR}_{t}=\left[\begin{array}{ccc}
\operatorname{SINR}_{\mathbf{t}}(1,1) & \cdots & \operatorname{SINR}_{\mathbf{t}}(1, K) \\
\vdots & \ddots & \vdots \\
\operatorname{SINR}_{\mathrm{t}}(L, 1) & \cdots & \operatorname{SINR}_{\mathrm{t}}(L, K)
\end{array}\right],
$$

where the columns indicate the carrier frequencies and the columns indicate the terrestrial links.
Regarding the inter-system interference, let us define $\mathbf{G}(l) \in$ $\mathbb{R}^{M \times K}$ as a matrix containing the interference level $g_{l}(m, k)$, which is defined as the interference caused by the $l$-th terrestrial link operating at $k$-th carrier and received at the $m$-th satellite link. The latter can be written as,

$$
g_{l}(m, k)=P_{\mathrm{tx}}^{\mathrm{TER}}(l) \cdot G_{\mathrm{tx}}^{\mathrm{TER}}\left(\theta_{l, m}\right) \cdot h(l, k, m) \cdot G_{\mathrm{rx}}^{\mathrm{SAT}}\left(\theta_{m, l}\right) .
$$

The information contained in $\mathbf{G}(l)$ is used to identify the satellite link that gets the highest level of interference when the $l$-th terrestrial link operates in carrier $k$. This is, for each $k$ th carrier, $M_{\mathrm{w}}(l, k)=\max _{m}[\mathbf{G}(l)]_{k}$, where $[\mathbf{G}(l)]_{k}$ denotes the $k$-th column of matrix $\mathbf{G}(l)$ and $M_{\mathrm{w}}(l, k)$ indicates the worst satellite link in terms of interference when terrestrial link $l$ operating in carrier $k$. For convenience, let us define the following matrix containing these worst satellite links as follows,

$$
\mathbf{M}_{\mathrm{w}}=\left[\begin{array}{ccc}
M_{\mathrm{w}}(1,1) & \cdots & M_{\mathrm{w}}(1, K) \\
\vdots & \ddots & \vdots \\
M_{\mathrm{w}}(L, 1) & \cdots & M_{\mathrm{w}}(L, K)
\end{array}\right] .
$$

Next, the SINR level of these worst satellite links is computed considering the satellite carrier allocation of Step 1 when only the $l$-th terrestrial link active. These SINR values are captured in the following matrix,

$$
\mathbf{S I N R}_{s}=\left[\begin{array}{ccc}
\operatorname{SINR}_{\mathrm{s}}(1,1) & \cdots & \operatorname{SINR}_{\mathrm{s}}(1, K) \\
\vdots & \ddots & \vdots \\
\operatorname{SINR}_{\mathrm{s}}(L, 1) & \cdots & \operatorname{SINR}_{\mathrm{s}}(L, K)
\end{array}\right],
$$

which captures the individual interference effect of each terrestrial link operating at the different carriers.

The two SINR matrices, namely SINR $_{t}$ and $\mathbf{S I N R}_{s}$, can be transformed into rate matrices, $\mathbf{R}_{t}$ and $\mathbf{R}_{s}$, by computing $\log _{2}(1+$ SINR $)$ of each matrix element. Finally, cost matrices can be obtained by replacing all values $\mathrm{R}_{\mathrm{x}}(l, k)$ with $\mathrm{R}_{\mathrm{x}}^{\max }-$ $\mathrm{R}_{\mathrm{x}}(l, k)$, where $\mathrm{R}_{\mathrm{x}}^{\max }=\max _{l, k} \mathrm{R}_{\mathrm{x}}(l, k)$, for $x=\{t, s\}$. The resulting cost matrices are named $\mathbf{C}_{t}$ and $\mathbf{C}_{s}$, respectively.

The proposed terrestrial assignment algorithm reduces to minimize the cost represented by these two matrices, one corresponding to the terrestrial segment performance and one corresponding to the satellite segment performance. The biobjective optimization problem can be reduced to a singleobjective optimization problem with the weighted sum technique in which the two cost matrices are combined in a single one by performing simple weighted addition, i.e. $w_{t} \mathbf{C}_{t}+w_{s} \mathbf{C}_{s}$. The resulting single objective assignment can be solved with the Hungarian method [16]. In this paper, the weights are all set to one so that no priority is given to either terrestrial or satellite links. Different weighting as well as more elaborated techniques for bi-objective optimization will be considered in future work.

The proposed algorithm for the terrestrial carrier assignment is summarized in Algorithm 1, which makes use of the previous ideas. Essentially, the proposed algorithm is based on a process that assigns all the available carriers to the best unassigned terrestrial links, in a single-carrier per link basis, according to $\mathbf{R}_{t}$ and $\mathbf{R}_{s}$ (steps 3 until 14 of Algorithm 


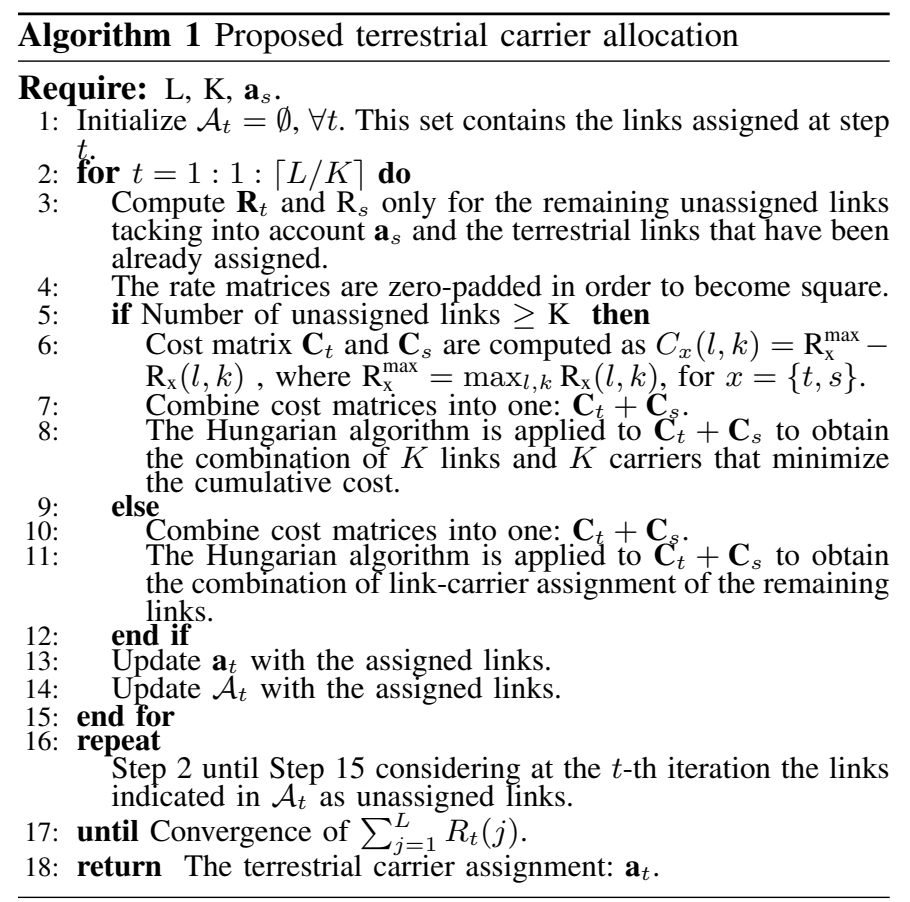

1). This process is repeated tacking into account previous assignments until all $L$ links are assigned (for loop in step 2 of Algorithm 1). Once all $L$ links have been assigned, the algorithm iterates over the previous procedure by considering the oldest assigned links as the unassigned links (steps 16 and 17 of of Algorithm 1). Note that for the computation of $\mathbf{R}_{t}$ and $\mathbf{R}_{s}$, only the links that have been already assigned are considered. Due to the sequential assignment considered in Algorithm 1, the algorithm is expected to take few iterations until the terrestrial sum-rate evolution converges to a steady state which corresponds to a local optimal solution of (7).

\section{Simulation Results}

In order to evaluate the proposed carrier allocation strategy, we consider the HSTB network topology depicted in Fig. 2, where we have $N=15$ terrestrial nodes that are interconnected via $L=28$ unidirectional communication links. The terrestrial topology shown in Fig. 2 is the same as the one in Fig. 1, which is a true backhaul topology being used in Finland. The complete database related to the terrestrial links have been obtained from FICORA, and this includes information listed on a station by station basis with the geographical location, maximum antenna gain, transmit power, channel bandwidth, etc. This information will be used here to properly model the interference levels using (2) and (5). The carrier allocation indicated in the database will be considered as the benchmark for comparison purposes. The database indicate that 8 carriers of $B_{t}=56 \mathrm{MHz}$ are used to allocate the $L=28$ terrestrial links. These carriers are divided into two blocks of 4 carriers each: one block from 17700 to $17924 \mathrm{MHz}$ and another block from 18708 and $18934 \mathrm{MHz}$.

With respect to the satellite segment, we assume that $M=3$ out of $N=15$ terrestrial nodes are equipped with a satellite

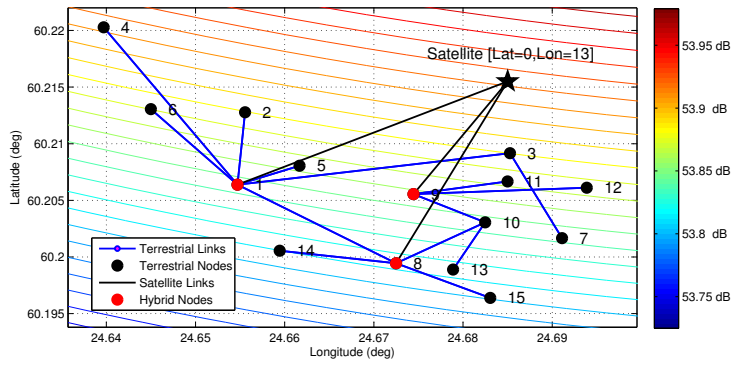

Fig. 2: Simulated HSTB topology with the simulated satellite beam pattern.

TABLE I: Simulation Parameters

\begin{tabular}{|c|c|}
\hline \multicolumn{2}{|c|}{ Terrestrial Segment } \\
\hline Parameter & Value \\
\hline \multirow{10}{*}{$\begin{array}{c}\text { No. available carriers }(K) \\
\text { Spanning frequency } \\
\text { Antenna pattern } \\
\text { Max. antenna gain }\left(G_{\mathrm{tx}}^{\mathrm{TER}}(0)\right) \\
\text { Transmit Power }\left(P_{\mathrm{tx}}^{\mathrm{TER}}(i)\right) \\
\text { Channel } \\
\text { Bandwidth }\left(B_{t}\right) \\
\text { Link distance } \\
\text { Noise power }\left(N_{t}\right) \\
\text { Antenna height } \\
\text { Terminal altitude above the sea level }\end{array}$} & $\begin{array}{c}8 \\
\end{array}$ \\
\hline & $\begin{array}{l}\text { from } 17700 \text { to } 17924 \mathrm{MHz} \\
\text { from } 18708 \text { to } 18934 \mathrm{MHz}\end{array}$ \\
\hline & ITU-R F.1245-2 \\
\hline & $38 \mathrm{dBi}$ \\
\hline & Between -26 and $-22 \mathrm{dBW}$ \\
\hline & $\begin{array}{l}\text { LoS channel (path and diffraction losses) } \\
\qquad 56 \mathrm{MHz}\end{array}$ \\
\hline & Between $427-1750 \mathrm{~m}$ \\
\hline & -121.52 dBW@B $B_{t}$ \\
\hline & Between $10-48 \mathrm{~m}$ \\
\hline & From terrain data available online \\
\hline \multicolumn{2}{|c|}{$\begin{array}{ll}\text { Satellite Segment } \\
\end{array}$} \\
\hline \multirow{3}{*}{$\begin{array}{c}\text { Parameter } \\
\text { No. available carriers }(C) \\
\text { Spanning frequency }\end{array}$} & Value \\
\hline & $6 \quad 6$ \\
\hline & from 17700 to $17887.2 \mathrm{MHz}$ \\
\hline \multirow{12}{*}{$\begin{array}{c}\text { Satellite location } \\
\text { Satellite antenna gain }\left(G_{s}\right) \\
\text { Dish antenna pattern } \\
\text { Max. dish antenna gain }\left(G_{\mathrm{rx}}^{\mathrm{SAT}}(0)\right) \\
\text { Carrier over co-channel interference } \\
\text { Transmit power }\left(P_{\mathrm{s}}\right) \\
\text { Channel } \\
\text { Bandwidth }\left(B_{s}\right) \\
\text { Link distance } \\
\text { Noise power }\left(N_{s}\right) \\
\text { Dish antenna height } \\
\text { Terminal altitude above the sea level }\end{array}$} & $13^{\circ} \mathrm{E}$ \\
\hline & Between $53.81-53.84 \mathrm{dBi}$ \\
\hline & ITU-R S.465 \\
\hline & $42.1 \mathrm{dBi}$ \\
\hline & $10.5354 \mathrm{~dB}$ \\
\hline & $9.23 \mathrm{dBW}$ \\
\hline & LoS channel (path loss) \\
\hline & $62.4 \mathrm{MHz}$ \\
\hline & $35.786 \mathrm{~km}$ \\
\hline & $-126.47 \mathrm{dBW} @ B_{s}$ \\
\hline & Half of the terrestrial height \\
\hline & From terrain data available online \\
\hline
\end{tabular}

dish antenna. The hybrid nodes, which are indicated with a red dot in Fig. 2, has been located assuming that nodes 1,8 and 9 will suffer traffic congestion due to the traffic aggregation from several links. We assume a multi-beam satellite located at orbital position $13^{\circ} \mathrm{E}$. The beam pattern has been simulated as in [17] and it is depicted as well in Fig. 2. A summary of the system parameters considered for the simulation set-up is given in Table I.

Table II and Table III shows the results obtained when the proposed algorithm is applied to the topology depicted in Fig. 2. The satellite assignment $\mathbf{a}_{s}$ resulting from Step 1 is fed into the second step of the proposed carrier allocation method in order to obtain the terrestrial carrier assignment. The results of the satellite assignment are summarized in Table II and the ones corresponding to the overall HSTB network are given in Table III. The sum-rate evolution of the terrestrial links resulting from Algorithm 1 is illustrated in Fig. 3. We also provide comparison with the terrestrial benchmark allocation found in the database in Table III.

The results shown in Fig. 3 reveal that the iterative terrestrial 


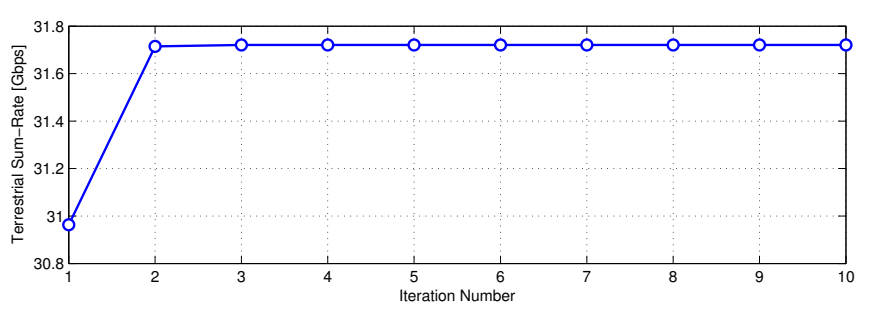

Fig. 3: Evolution of the terrestrial sum-rate obtained with Algorithm 1.

TABLE II: Results after Step 1

\begin{tabular}{|c|c|}
\hline & Satellite Segment \\
\hline Satellite assignment $\left(\mathbf{a}_{s}\right)$ & {$[2,3,1]$} \\
Num. of Carriers (C') & 3 \\
Spectrum used [MHz] & 187.2 \\
mean SINR [dB] & 10.30 \\
(no interference from terrestrial yet) & 664.70 \\
Sum-Rate [Mbps] & 3.55 \\
SE [bps/Hz] & \\
\hline
\end{tabular}

TABLE III: Results after Step 2

\begin{tabular}{|c|c|c|}
\hline & Terrestrial Segment & Terrestrial Benchmark \\
\hline Terrestrial assignment $\left(\mathbf{a}_{t}\right)$ & {$[4,5,8,7,6,2,1,3,4,5,1,3,3,1, \ldots$} & {$[8,4,7,3,7,3,6,2,6,2,6,2,1,5, \ldots$} \\
\hline Num. of Carriers (K') & $1,1,3, J, 0,2,4, I, 0,0, \Xi, 0,4, I, 2]$ & $\stackrel{4,8,3,1,2,6,2,6,4,8,4,8,4,8]}{8}$ \\
\hline Spectrum used [MHz] & 448 & 448 \\
\hline mean SINR [dB] & 60.90 & 56.04 \\
\hline Sum-Rate [Gbps] & 31.721 & 29.190 \\
\hline $\mathrm{SE}[\mathrm{bps} / \mathrm{Hz}]$ & 70.81 & 65.16 \\
\hline & \multicolumn{2}{|c|}{ Satellite Segment } \\
\hline Terrestrial assignment $\left(\mathbf{a}_{s}\right)$ & \multicolumn{2}{|c|}{ same as Table II } \\
\hline Num. of Carriers (C') & \multicolumn{2}{|c|}{$\begin{array}{l}\text { same as Table II } \\
\text { same as Table II }\end{array}$} \\
\hline Spectrum used [MHz] & \multirow{2}{*}{\multicolumn{2}{|c|}{$\begin{array}{c}\text { same as Table II } \\
5.28\end{array}$}} \\
\hline mean SINR [dB] & & \\
\hline $\begin{array}{l}\text { Sum-Rate [Mbps] } \\
\text { SE [bps/Hz] }\end{array}$ & \multicolumn{2}{|c|}{489.97} \\
\hline & \multicolumn{2}{|c|}{ HSTB network } \\
\hline Spectrum used $[\mathrm{MHz}]$ & \multicolumn{2}{|c|}{448} \\
\hline Sum-Rate [Gbps] & \multirow{2}{*}{\multicolumn{2}{|c|}{$\begin{array}{l}32.211 \\
71.90\end{array}$}} \\
\hline SE [bps/Hz] & & \\
\hline
\end{tabular}

carrier allocation algorithm converges after only 2 iterations to a stable value of $31.721 \mathrm{Gbps}$, which is higher than that the $29.190 \mathrm{Gbps}$ provided by the benchmark allocation. From Table III, it can be observed that, as expected, the terrestrial segment tend to make use of as much spectrum as possible in order to avoid interference scenarios and, thus, maximize the sum-rate. Once the terrestrial segment is assigned, the sum-rate of the satellite segment is deteriorated due to the terrestrial interference going from $664.70 \mathrm{Mbps}$ to 489.97 Mbps. By looking at the HSTB network overall numbers, it can be observed that considering the integration of the satellite segment with the conventional terrestrial backhaul network topology together with the proposed joint carrier allocation optimization resulted in an approximately $10 \%$ spectral efficiency improvement with respect to the benchmark carrier allocation of the terrestrial only backhaul network.

\section{Conclusion}

In this paper, we proposed an efficient carrier assignment approach to solve the radio resource sharing problem in HSTB networks, where the satellite segment and the terrestrial backhaul operate in the same 17.7-19.7 GHz band. Given the intractability of the problem, we proposed to solve the joint optimization by first optimizing the satellite carrier allocation and, in a next step, optimize the terrestrial network subject to the previous satellite carrier assignment. Results based on computer simulations were presented, which showed that the conventional terrestrial backhual networks can be improved by considering the satellite segment together with the proposed carrier allocation approach.

\section{ACKNOWLEDGMENT}

This work was partially supported by European Commission in the framework of the H2020 SANSA project (Grant agreement no. 645047) and by the National Research Fund, Luxembourg, under CORE project SeMIGod and CORE project SATSENT.

\section{REFERENCES}

[1] A. Gupta and R.K. Jha, "A Survey of 5G Network: Architecture and Emerging Technologies," IEEE Access, vol. 3, pp. 1206-1232, Aug. 2015.

[2] X. Ge, H. Cheng, M. Guizani, and T. Han, "5G Wireless Backhaul Networks: Challenges and Research Advances," IEEE Network, vol. 28, no. 6, pp. 6-11, Dec. 2014.

[3] E. Hossain and M. Hasan, "5G Cellular: Key Enabling Technologies and Research Challenges," IEEE Instrum. Meas. Mag., vol. 18, no. 3 , pp. 11-21, Jun. 2015.

[4] H.S. Dhillon and G. Caire, "Wireless Backhaul Networks: Capacity Bounds, Scalability Analysis and Design Guidelines," IEEE Trans. Wireless Commun., vol. 14, no. 11, pp. 6043-6056, Jun. 2015.

[5] European Commission, "Digital Agenda for Europe," http://ec.europa. eu/digital-agenda/digital-agendaeurope, 2016, [Online].

[6] T. De Cola, D. Tarchi, and A. Vanelli-Coralli, "Future Trends in Broadband Satellite Communications: Information Centric Networks and Enabling Technologies," International Journal of Satellite Communications and Networking, Apr. 2015.

[7] H2020 VITAL project, "VIrtualized hybrid satellite-TerrestriAl systems for resilient and fLexible future networks," http://ict-vital.eu, 2015-2017.

[8] H2020 SANSA project, "Shared Access terrestrial-satellite backhaul Network enabled by Smart Antennas," http://sansa-h2020.eu, 20152018.

[9] S. Chatzinotas et al., "Spectrum Awareness and Resource Allocation Techniques for Satellite Cognitive Radio Networks," Int. J. Sat. Commmunications and Networking., to appear, 2016.

[10] E. Lagunas, S.K. Sharma, S. Maleki, S. Chatzinotas, and B. Ottersten, "Resource Allocation for Cognitive Satellite Communications with Incumbent Terrestrial Networks," IEEE Trans. On Cognitive Communications and Networking, vol. 1, no. 3, pp. 305-317, Nov. 2015.

[11] L. Rosati and G. Reali, "Jointly Optimal Routing and Resource Allocation in Hybrid Satellite/Terrestrial Networks," Int. Workshop in Satellite and Space Communications, Madrid, Spain, Sept. 2006.

[12] L. Xiao, M. Johansson, and S.P. Boyd, "Simultaneous Routing and Resource Allocation via Dual Decomposition," IEEE Trans. Commun., vol. 52, no. 7, pp. 1136-1144, Jul. 2014.

[13] M. Cao, X. Wang, S.J. Kim, and M. Madihian, "Multi-Hop Wireless Backhaul Networks: A Cross-Layer Design Paradigm," IEEE J. Sel. Areas Commun., vol. 25, no. 4, pp. 738-748, May 2007.

[14] Z.Q. Luo and S. Zhang, "Dynamic Spectrum Management: Complexity and Duality," IEEE J. Sel. Topics Signal Process., vol. 2, no. 1, pp. 57-73, Feb. 2008.

[15] S.K. Sharma, E. Lagunas, S. Maleki, S. Chatzinotas, J. Grotz, J. Krause, and B. Ottersten, "Resource Allocation for Cognitive Satellite Communications in Ka-band (17.7-19.7 GHz)," IEEE Int. Conf. on Communications (ICC), London, UK, Jun. 2015.

[16] H.W. Kuhn, "The Hungarian Method for the Assignment Problem," Naval Research Logistics Quarterly, vol. 2, pp. 83-97, 1955.

[17] C. Caini, G.E. Corazza, G. Falciasecca, M. Ruggieri, and F. Vatalaro, "A Spectrum and Power Efficient EHF Mobile Satellite System to be Integrated with Terrestrial Cellular Systems," IEEE J. Sel. Areas Commun., vol. 10, no. 8, pp. 1315-1325, 1992. 